\title{
Cosmic Star Formation History and Deep Field X-ray Studies
}

\author{
Pranab Ghosh ${ }^{1}$ \\ ${ }^{1}$ Tata Institute of Fundamental Research \\ Bombay 400 005, India \\ email: pranab@tifr.res.in
}

\begin{abstract}
We explore the X-ray diagnostics of cosmic star formation history that have possible form recent deep field X-ray studies. We summarize the status of our understanding of the X-ray evolution of galaxies. We indicate the lessons learnt so far from number count plots, and criteria for distinguishing between normal/straburst galaxies and AGNs in deep X-ray surveys. We summarize how the observed correlations between X-ray emission and that at other wavebands indicate the value of $\mathrm{X}$-rays as a probe of cosmic star formation.
\end{abstract}

Keywords. stars: formation, X-rays: galaxies, X-rays: binaries, infrared: galaxies, submillimeter, galaxies: starburst.

\section{Introduction}

We discuss in this paper deep-field X-ray studies done in recent years, particularly the Chandra Deep Field North and South (CDF-N/S) surveys, and how they bear on the subject of cosmic star-formation history. We summarize those aspects of the observations which we most need for this purpose, and indicate the status of our understanding in these various aspects, as also in this subject as a whole. We discuss first how the X-ray luminosities of normal and starburst galaxies evolve with redshift, and the role of cosmic star-formation history in this. Next, we indicate what diagnostics have been available from the $\log N-\log S$ plots in the X-rays from these deep surveys that bear on this question. A major issue in this subject has been the relative roles of normal/starburst galaxies on the one hand, and active galactic nuclei (AGNs) on the other, and we discuss suggested criteria for distinguishing between them. Finally, the correlations between Xray properties and those in other wavebands - optical, infrared, submillimeter, and radio - are proving to be of much diagnostic value, and we summarize those points about them that bear directly on star formation.

\section{Evolution of X-ray Luminosity}

With exposures of duration $\sim$ megaseconds (Ms) with the Chandra observatory, statistical studies of the X-ray evolution of normal, starburst, and Lyman break galaxies became possible in the early 2000s, and was pioneered by several groups, particularly the one at Pennsylvania State University (Brandt et al. (2001a); Hornschemeier et al. (2002); Lehmer et al. (2005)). The technique used was that of stacking, wherein X-ray photons from a collection of optically well-known galaxies in the field of exposure, which were not detected individually in the X-rays, were summed together, i.e., stacked, and analyzed to obtain an average description of a typical galaxy in the collection. This gave us the first indication of how X-ray luminosities of galaxies, $L_{x}$, have evolved with the redshift $z$. It became possible to study the evolution of $L_{x}$ with $z$ for normal/starburst 


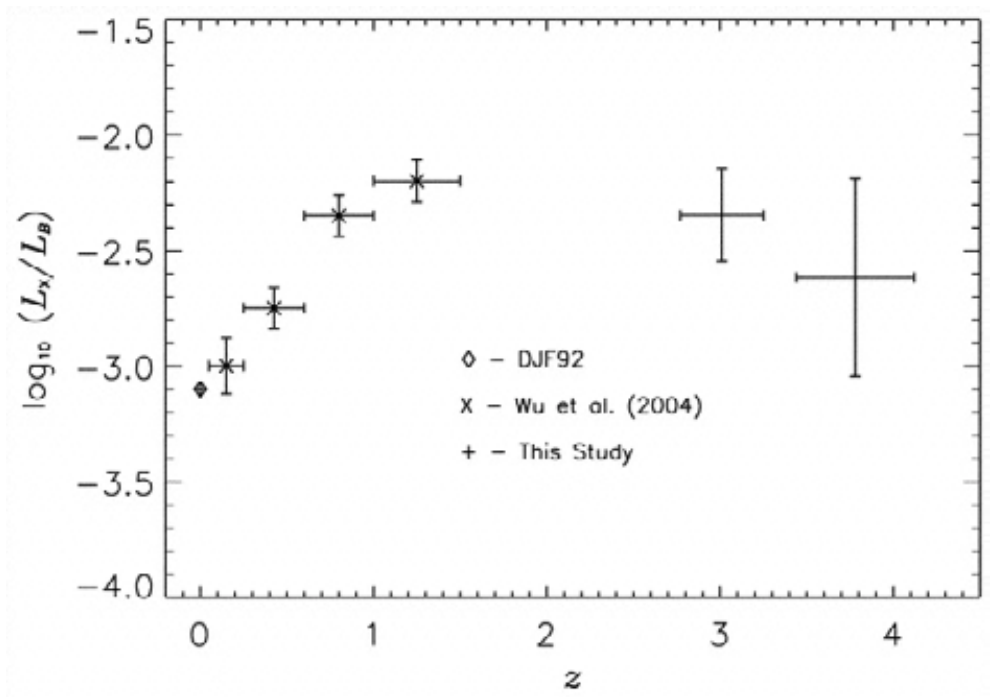

Figure 1. X-ray $(0.5-4.5 \mathrm{keV})$ to B-band luminosity ratio vs. redshift. Reproduced by permission from Lehmer et al. (2005). Sources of data given in original reference.

galaxies at relatively low redshifts, $z \sim 0 \rightarrow 1$, as also the $L_{x}$-evolution of Lyman-break galaxies, which are believed to be distant analogs of local starburst galaxies, at intermediate redshifts $z \sim 2 \rightarrow 4$. The current observational situation is shown in Fig. 1, taken from Lehmer et al. (2005). Note that what is displayed is actually $L_{x} / L_{B}$, the ratio of the X-ray luminosity to the B-band luminosity. The basic result is clear: $L_{x} / L_{B}$ rises by a factor $\sim 10$ as we look back from the local universe $(z \approx 0)$ to a redshift $\sim 1$, and falls somewhat as $z$ increases from $\sim 3$ to $\sim 4$. The former result comes from stacked normal galaxies, and the latter from stacked Lyman break galaxies, in CDF-N ( $\sim 2$ Ms exposure) and CDF-S $\left(\sim 1\right.$ Ms exposure). The expected peak in the evolution of $L_{x} / L_{B}$ is therefore in the range $z \sim 1.5 \rightarrow 3$.

\subsection{Cosmic Star Formation History}

Research on the connection between cosmic star-formation history and $L_{x}$-evolution of normal galaxies was introduced by White \& Ghosh (1998) and Ghosh \& White (2001), the basic idea being as follows. Since galaxies of the above type do not harbor AGNs normally, or any low-luminosity AGNs present do not dominate the X-ray emission, their total X-ray output must be the integrated output of their X-ray binaries, supernova remnants, and hot gas. Focusing their attention on X-ray binaries, these authors argued that the evolution of the X-ray binary populations in a galaxy must be determined by its star-formation history, since these stars evolve and produce the compact objects in interacting binaries that generate X-rays by accretion from the binary companion. Ghosh \& White (2001) developed a scheme for calculating the evolution of the X-ray binary population and the X-ray luminosity $L_{x}$ of a galaxy, given its star formation history (SFH). For the average description of a collection of galaxies, a suitable prescription for the cosmic star formation history is appropriate, and these authors used a range of cosmic SFHs that cover the possibilities, given in the literature. The results for four representative cosmic SFHs are shown in Fig. 2, taken from Ghosh \& White (2001). These SFHs are (a) the original Madau profile, (b) the "anvil" profile, corresponding to a monolithic scenario of star formation, (c) the hierarchical profile, in which stars form in bursts during hierarchical merging of structures, and (d) the gaussian profile, wherein 

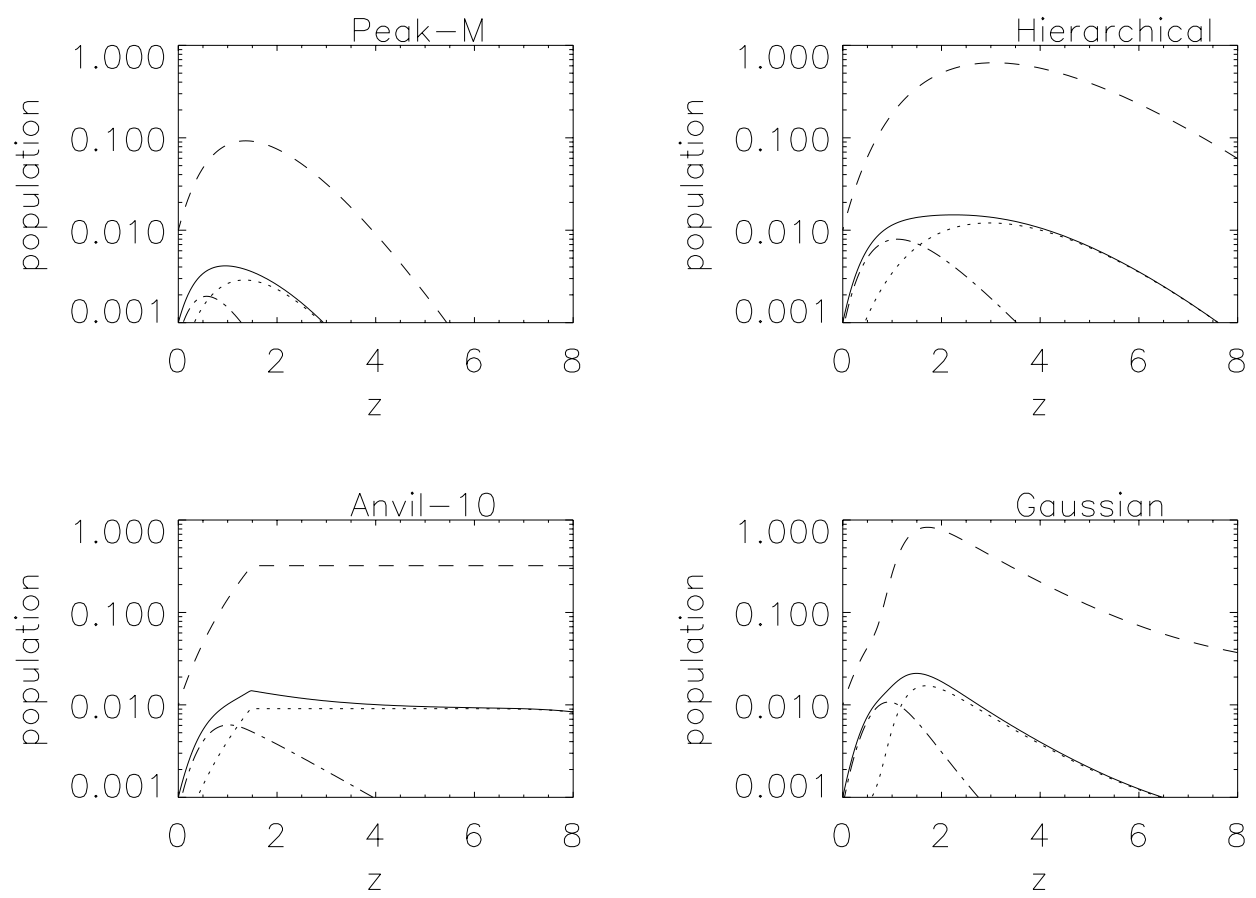

Figure 2. Evolution of X-ray binary populations and total X-ray luminosities from Ghosh and White (2001). Shown are evolutions for four cosmic star formation histories (SFH) that cover the current range of possibilities. Lines coded as follows:

---- Cosmic SFH $\quad \cdots \cdot$ HMXBs - - LMXBs $\quad$ Total $L_{x}$

a gaussian component is added at intermediate $z$ to a Madau-type profile, in order to account for the intense, dust-enshrouded star formation at these redshits, as detected at submm wavelengths (Blain et al. (1999)).

The massive X-ray binaries (HMXBs) evolve promptly, on timescales of a few million years to $\sim 10^{7}$ years, following the SFH closely. By contrast, the low-mass X-ray binaries (LMXBs) evolve much more slowly, on overall timescales of a few billion years (Gyr), and so follow with a considerable time lag, as shown in Fig. 2. (Note that this LMXB lag consists of two parts: after the neutron star is produced in the supernova of a Hestar, the post-supernova binary takes $\tau_{P S N B} \sim 2$ Gyr to become a mass-transferring LMXB, which then evolves on a timescale $\tau_{L M X B} \sim 1$ Gyr or so. See White \& Ghosh (1998).) The profile of the total X-ray luminosity $L_{x}$ therefore carries signatures of the SFH, different SFHs yielding different $L_{x}$-evolution profiles. Consequently, observed $L_{x^{-}}$ profiles can ultimately constrain SFHs.

Comparing Figs. 1 and 2, it is clear that the predictions from cosmic SFHs are qualitatively correct, except possibly in the anvil case. Further, the hierarchical and gaussian profiles appear to give better agreement than the original Madau profile. For more quantitative understanding, we must first clarify the observed and calculated quantities. Theory tells us that, for the choices of $\tau_{P S N B}$ and $\tau_{L M X B}$ made above, $L_{x}$ rises by a factor $\sim 10$ as $z$ increases from 0 to 1 . This means that $L_{x} / L_{B}$ would also rise by the same factor only if $L_{B}$ had little evolution in the same redshift range. Observations tell us that $L_{x} / L_{B}$ rises by $\sim 10$ as $z$ increses from 0 to 1 . The question therefore is: by what factor does the observed $L_{x}$ rise in this range of $z$ ? The answer is not quite clear yet. With 


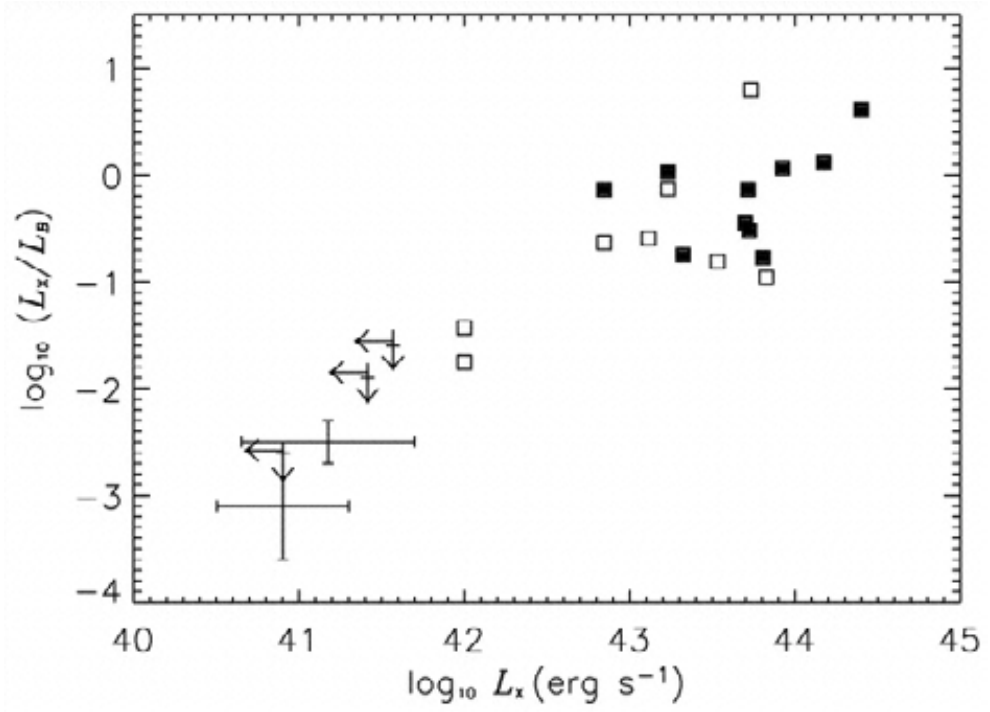

Figure 3. $L_{x} / L_{B}$ vs. $L_{x}$ for stacked galaxies (plus signs) and individually detected sources (open squares) in CDF, the latter being almost all AGNs. For comparison, known broad-line AGNs are also plotted (filled squares) Reproduced by permission from Lehmer et al. (2005).

the 0.5 Ms CDF-N exposure, the original Brandt et al. (2001a) result was that $L_{x}$ rose by a factor $\sim 3$ in this redshift range, which was consistent with the subsequent result of Hornschemeier et al. (2002) with the greater $\sim 1$ Ms CDF-N exposure. The corresponding result from the current $\sim 2 \mathrm{Ms}$ exposure needs to be clarified.

\subsection{Understanding $L_{x}$-Evolution}

If we assume that $L_{x}$ does rise by $\sim 3$ as $z \sim 0 \rightarrow 1$, then there are several issues to be addressed. First, with the rise of $L_{x} / L_{B}$ by $\sim 10$, this implies that $L_{B}$ would be falling by a factor of $\sim 3$ as $L_{x}$ rises in this redshift range. How do we understand this? In fact, the situation is brought out very instructively in Fig. 3, also reproduced from Lehmer et al. (2005), where $L_{x} / L_{B}$ is shown against $L_{x}$. The correlation between them is such that $L_{x}$ does, indeed, rise by about a factor of 3 in this redshift range. Hence, this apparent fall in $L_{B}$ remains to be understood, since our experience in local universe is that $L_{x}$ correlates positively with $L_{B}$. Figure 3 also shows the interesting behavior of individually detected sources in CDF at $z \sim 2-4$, which consist almost entirely of AGNs: these have much higher values of $L_{x} / L_{B}$, ranging from $\sim 0.1$ to a few, while the stacked galaxies, normal, starburst, or Lyman break, have much lower values, $L_{x} / L_{B} \sim 10^{-2}-10^{-3}$. This ratio should thus be a good discriminator between galaxies and AGNs, a point to which we return below.

If the above observed rise in $L_{x}$ by $\sim 3$ as $z \sim 0 \rightarrow 1$ is borne out by further work, how would we reconcile it with the rise by $\sim 10$ given in the same redshift range by the evolutionary calculations of Fig. 3 with the above choice of parameters? We consider two possibilities. An obvious solution would be that the evolutionary timescale $\tau_{L M X B}$ of LMXBs is longer than we used above, say, $\sim 2$ Gyr. This would change the rise-factor in evolutionary calculations to about 3 because, with longer lag, the peak of the LMXB emission would occur closer to $z=0$, so that the fall from the peak to the value at the present epoch would not be quite so large. While such a value is not impossible, it appears higher than the canonical values adopted currently. An interesting alternative is the bandpass-change factor, as used in the standard expression used for converting X-ray 
fluxes $f_{x}$ to X-ray luminosities $L_{x}$, namely,

$$
L_{x}=4 \pi d_{L}^{2} f_{X}(1+z)^{\Gamma-2},
$$

may not be quite right. The bandpass-change factor $(1+z)^{\Gamma-2}$ in the above equation takes account of the fact that what we observe in the Chandra band is, in fact, what the source at a redshift $z$ emits in a higher energy-band in its own rest-frame, the connection involving the photon index $\Gamma$ of the source spectrum. It is customary to use $\Gamma=2$ from the fits made X-ray spectra of galaxies in the $1-10 \mathrm{keV}$ band. However, we argued above that, in the $0<z<1$ range, $L_{x}$ is dominated by LMXBs, and it is well-known that LMXB spectra have soft excesses, i.e., extra emission at soft X-rays at $<1 \mathrm{keV}$ over and above the 1-10 keV trend. Recent XMM-Newton spectra of LMXBs bear this out fully (Sidoli et al. (2005)). So, if we make an extrapolation of the $1-10 \mathrm{keV}$ trend to the Chandra soft band, we shall be underestimating $L_{x}$ at redshifts $\sim 0.5-1$. Rough estimates from LMXB spectra show that this effect can, indeed, lead to underestimates by factors $\sim 3$ in the rise of $L_{x}$ in the above redshift range. The effect needs to be studied in more detail.

\section{X-ray $\log N-\log S$ diagnostics}

Source counts, i.e., $\log N-\log S$ plots, from the deep X-ray surveys are determining the contributions of the various faint X-ray source populations to the extragalactic X-ray background (XRB). With $\sim 89 \%$ the XRB resolved in the CDF in the Chandra soft band $(0.5-2 \mathrm{keV})$, and $\sim 93 \%$ resolved in the hard band $(2-8 \mathrm{keV})$, the contributions from (a) bright, unobscured AGNs, (b) obscured AGNs, and (c) normal and starburst galaxies are showing their characteristic signatures (Bauer et al. (2004)). The overall slope of the $\log N-\log S$ plot in both soft and hard bands is $\sim 0.55$. AGNs have a flat slope $\sim 0.48$, rather similar to the overall one. But normal galaxies have a much steeper overall slope of $\sim 1.3$. Of these, quiescent galaxies have a slope $\sim 1.3$ like the total, ellipticals flatter at $\sim 1.1$, and starbursts much steeper at $\sim 1.7$. It is clear, therefore, that the number counts in both soft and hard bands will become dominated by normal galaxies at sufficiently low fluxes. This critical flux is estimated to be $\sim 10^{-17} \mathrm{erg} \mathrm{cm}^{-2} \mathrm{~s}^{-1}$ in the soft band (Bauer et al. (2004)).

\subsection{Normal/Starburst Galaxies vs. AGNs}

How do we distinguish between normal/starburst galaxies and AGNs? This is an important question, since the interplay between these two components of the XRB and the number-count plots plays an essential role in our understanding. We now consider some of the most useful discriminators that are being utilized for this purpose:

- X-ray luminosity $L_{x}$. AGNs are generally brighter than normal/straburst galaxies, the critical value of $L_{x}$ in the Chandra full band $(0.5-8 \mathrm{keV})$ being $\sim 3 \times 10^{42} \mathrm{erg} \mathrm{s}^{-1}$.

- X-ray spectra. AGN spectra are harder than those of normal galaxies. In terms of a hardness ratio, i.e., that between hard- and soft-band fluxes, the critical value is $\sim 0.8$. The corresponding critical photon index is $\Gamma \sim 1$.

- X-ray to optical flux ratio, $f_{x} / f_{\text {opt }}$, the X-ray flux being over the full band. AGNs have a higher value than than normal/starburst galaxies (see Fig. 3 ). The critical value $f_{x} / f_{\text {opt }} \approx 0.1$ is one of the most useful discriminators.

- Optical spectra. AGNs display characteristic broad/ high-ionization emission lines.

- Radio properties.

The collection of X-ray discriminators can be conveniently displayed in a plot of Rband magnitude vs. X-ray flux, as been done by Bauer et al. (2004) in their Fig. 7. The 
criteria (the first three in the above list) work well together, the $f_{x} / f_{\text {opt }} \approx 0.1$ criterion being particularly useful, and a fairly effective separation of AGNs and normal galaxies is possible on such plots. The latest version of such a plot is given by Hornschemeier (these proceedings).

The results from such separation of AGN and galaxy contributions to the XRB and the number-count plots is as follows. The power in the XRB is certainly dominated by AGNs, as $85-90 \%$ of it comes from AGNs, and only 5-15\% comes from normal/starburst galaxies. Of the latter, starbursts dominate in the soft band, and quiescents dominate in the hard band. However, in number density, star-forming galaxies overtake AGNs, and dominate the sky, at faint fluxes given above, the threshold corresponding to a factor of a few below the current CDF soft-band flux limit (Bauer et al. (2004)).

\section{Correlations Between X-rays and Other Wavebands}

These correlations are shedding more light on star formation in distant galaxies, and we summarize the essential points here.

\subsection{X-ray/Optical Correlations}

The recent study of optically bright, X-ray faint (OBXF) galaxies in CDF-N has proved to be of much value in establishing the continuity between local starburst galaxies and Lyman break galaxies at intermediate redshifts, and so the viability of the whole picture given above (Hornschemeier et al. (2003)). These galaxies have low values of X-ray to optical flux ratios, $f_{x} / f_{R} \sim 0.01$ or less, and normal optical spectra, and so appear to be "distant analogs of 'normal' galaxies in the local universe" (Hornschemeier et al. (2003)), the redshift range being $z \sim 0.1-0.8$. OBXF appear to be dominated entirely by nonAGN processes, although some low-luminosity AGN activity cannot be ruled out. Their $\mathrm{X}$-ray luminosities exceed those of normal galaxies, however, and they have soft X-ray spectra, reminiscent of starbursts. In addition, their $\log N-\log S$ slope is very steep, $\sim 1.7$, almost identical to those of starbursts. Hence they are believed to be starburst at moderate redshifts, bridging the gap between local starbursts and the Lyman break galaxies at intermediate redshifts $2-4$.

\subsection{X-ray/IR Correlations}

There is a tight correlation between X-ray and $15 \mu \mathrm{m}$ infrared galaxy populations, the former coming from CDF-N and the latter from ISOCAM survey of the Hubble Deep Field North (Alexander et al. (2002)). These are luminous IR starburst galaxies, undergoing dust-enshrouded star formation. With small X-ray to infrared flux ratios, $f_{x} / f_{I R} \sim 0.03$ or less, these appear to be non-AGN sources. They are probably X-ray detected starburst galaxies. The key point is that, since $15 \mu \mathrm{m}$ emission is believed to be a good indicator of star formation, this correlation strongly suggests that so are X-rays.

\subsection{X-ray/Submm Correlations}

While seven out of ten of bright $850 \mu \mathrm{m}$ SCUBA sources have shown X-ray emission, five of these seven are AGNs (Alexander et al. (2003)), and they appear to show an anticorrelation between X-rays and $850 \mu \mathrm{m}$ emission, which is poorly understood. The key point here, however, is that AGNs contribute negligibly to submm emission, which must, therefore, be powered by star formation.

\subsection{X-ray/Radio Correlations}

The fact that there is a large overlap between CDF-N X-ray sources and $1.4 \mathrm{GHz}$ radio sources in this field in the VLA surveys, and that these sources show an excellent 
correlation between X-ray luminosity and radio luminosity, has established a close connection beyond doubt (Bauer et al. (2002)). What is particularly remarkable is that this correlation is the same at moderate redshifts as it is in the local universe. As radio emission is believed to be a good indicator of star formation, this again emphasizes the role of X-rays as indicators of star formation.

\section{Conclusions}

Our understanding of the X-ray evolution of normal/starburst galaxies appears to be qualitatively correct, but details need to clarified, both on observational issues, and on the development of a more sophisticated theory. While the power in XRB is dominated by AGNs, source number counts are expected to be dominated by star-forming galaxies at faint fluxes. Finally, correlations between X-ray emission and that in other wavebands stress the diagnostic value of X-rays in probing cosmic star formation.

\section{Acknowledgements}

It is a pleasure to acknowledge an IAU grant.

\section{References}

Alexander, D. M., et al. 2002, ApJ (Letters) 568, L85

Alexander, D. M., et al. 2003, AJ 125, 383

Bauer, F. E., et al. 2002, AJ 124, 2351

Bauer, F. E., et al. 2004, AJ 128, 2048

Blain, A. W., et al. 1999, MNRAS 302, 632

Brandt, W. N., et al. 2001a, AJ 122, 1

Brandt, W. N., et al. 2001b, ApJ (Letters) 558, L5

Ghosh, P. \& White, N.E. 2001, ApJ (Letters) 559, L97

Hornschemeier, A. E., et al. 2002, ApJ 568, 82

Hornschemeier, A. E., et al. 2003, AJ 126, 575

Lehmer, B. D., et al. 2005, AJ 129, 1

Sidoli, L., Parmar, A. N., \& Oosterbroek, T. 2005 A $\& A$ 429, 291

White, N.E. \& Ghosh, P. 1998, ApJ (Letters) 504, L31

\section{Discussion}

ZEZAS: The peak of the $\mathrm{L}_{X} / \mathrm{L}_{B}$ ratio seems to be around $\mathrm{z} \sim 1$ where the star-formation rate also peaks. However, at that point I would also expect to have a very strong contribution of HMXBs and ULXs which would result in a non-linear $\mathrm{L}_{X} / \mathrm{L}_{B}$ ratio.

GHosh: There is no doubt that $\mathrm{L}_{X} / \mathrm{L}_{B}$ is expected to be non-linear (i.e., $\mathrm{L}_{X} \propto \mathrm{L}_{B}{ }^{n}$, with $n \neq 1$ ), in general. In fact, values of $n>1$ are sometimes suggested in literature. So, I completely agree with you. The point would be to see how $\mathrm{n}$ changes with $\mathrm{z}$, to study the effects you are describing.

HoRnsCHEMEIER: Evolution in $\mathrm{L}_{B}$ is actually often due to observational selection. One has to be very careful about how you select galaxies so I would not over-interpret implied evolution in $\mathrm{L}_{B}$.

Ghosh: Yes, observers must advise us on the best ways of exploring $\mathrm{L}_{B}$ evolution. 